\title{
Comparison of various adhesion contact theories and the influence of dimensionless load parameter
}

\author{
XINGHUA SHI and YA-PU ZHAO* \\ State Key Laboratory of Nonlinear Mechanics (LNM), Institute of Mechanics, \\ Chinese Academy of Sciences, Beijing 100080, China
}

Received in final form 19 October 2003

\begin{abstract}
Three models, JKR (Johnson, Kendall and Roberts), DMT (Derjaguin, Muller, and Toporov) and MD (Maugis-Dugdale), are compared with the Hertz model in dealing with nano-contact problems. It has been shown that both the dimensionless load parameter, $\bar{P}=P /(\pi \Delta \gamma R)$, and the transition parameter have significant influences on the contact area at micro/nano-scale and should not be ignored in nano-indentation tests.
\end{abstract}

Keywords: Work of adhesion; elastic contact models; transition parameter; dimensionless load parameter.

\section{INTRODUCTION}

In recent years there has been considerable interest in the mechanical characterization of thin-film systems and small volumes of materials using depth-sensing indentation tests which utilize either spherical or pyramidal indenters $[1,2]$. Usually, to obtain values for hardness and elastic modulus of the specimen material from the experimental values of indenter load and depth of penetration is the principal goal of such testing. The forces involved are usually in the millinewton $\left(10^{-3} \mathrm{~N}\right)$ range and are measured with a resolution of a few nanonewtons $\left(10^{-9} \mathrm{~N}\right)$, and the depths of penetration are in the order of nanometers, hence the term "nano-indentation" $\left(10^{-9} \mathrm{~m}\right)$. As the experimental values of indenter load and depth of penetration give an indirect measure of the area of contact, from which the mean contact pressure, and thus hardness, can be estimated, the relationship between the contact area and the load is considerably important. Thus the appropriate use of the corresponding theoretical model will play a key role in the experimental investigation.

\footnotetext{
*To whom correspondence should be addressed. Tel.: (86-10) 6265-8008. Fax: (86-10) 6256-1284. E-mail: yzhao@lnm.imech.ac.cn
} 
Nano-contact mechanics refers to contact mechanics at nano-scales, which is fundamentally important for understanding of the force-distance curves from various scanning microscopies (atomic force microscopy (AFM), magnetic force microscopy (MFM), etc.) and of nano-indentation, the adhesion (or stiction) of microelectromechanical systems (MEMS) and nano-electromechanical systems (NEMS), nano-tribology and nano-wear. The emphasis of the present paper is to compare different adhesion contact mechanics models in their dimensionless form, and to discuss the influences of the governing dimensionless parameters.

\section{MODELS OF CONTINUUM ADHESION CONTACT MECHANICS}

Continuum models that predict the contact area for various geometries have a long history, dating back to the pioneering work of Hertz in 1881 [3]. Many of the most important contact problems are summarized in the book by Johnson in 1987 [4]. Hertz found that the radius of the circle of contact $a_{\mathrm{H}}$ was related to the indenter load $P$, the spherical indenter radius $R$, and the elastic properties of the contacting materials by:

$$
a_{\mathrm{H}}^{3}=\frac{P R}{K},
$$

where $K$ is the equivalent elastic modulus of the indenter tip and the sample, given by

$$
K=\frac{4}{3}\left(\frac{1-v_{1}^{2}}{E_{1}}+\frac{1-v_{2}^{2}}{E_{2}}\right)^{-1},
$$

where $E$ and $v$ are the Young's modulus and the Poisson's ratio, respectively, and subscripts 1 and 2 denote the indenter and the sample, respectively. If the contacting bodies are spheres with radii $R_{1}$ and $R_{2}$, then $R$ in the above equation is the equivalent radius given by: $R=R_{1} R_{2} /\left(R_{1}+R_{2}\right)$. In the absence of adhesion, the Hertz model has been shown to accurately describe the contact area between elastic bodies [4]. However, a great many experimental and theoretical results show that the surface-to-bulk ratio becomes significant at small scales [5]. Therefore, adhesion arising from attractive surface forces is generally not negligible and must be included in any description of contact area. Actually, with the increasing popularity of nanoscale technology and the increasing sensitivity of nano-indentation instruments, experimental results increasingly show that the contact area of the bodies is much larger than estimated with the Hertz model; especially, when the load diminishes to zero, the contact area reaches a constant value [4-8]. It proves that the surface forces, especially the adhesion force, do play an important role in the contact of the indenter and the sample at sub-micro/nano-scale.

Considering the contact between two rigid spheres with radii $R_{1}$ and $R_{2}$, the adhesion force, $P_{\mathrm{A}}$, between them is given by the Bradley theory [9] as

$$
P_{\mathrm{A}}=2 \pi \Delta \gamma R
$$


where $\Delta \gamma=\gamma_{1}+\gamma_{2}-\gamma_{12}$ is the work of adhesion, $\gamma_{1}$ and $\gamma_{2}$ are the surface energies of materials of spheres 1 and 2 , respectively, and $\gamma_{12}$, is the interfacial energy. Equation (2) applies to the case of rigid spheres in contact, i.e., the contact radius is zero. In practice, the spheres deform when placed in contact due to their finite value of elastic modulus. Derjaguin, Muller and Toporov [10] considered the case of deformable bodies by adding the force given by equation (2) to the Hertz contact equation and the resulting contact theory is referred to as the DMT theory. The DMT model gives the contact radius $a_{\mathrm{DMT}}$ related to the work of adhesion, $\Delta \gamma$, by:

$$
a_{\mathrm{DMT}}^{3}=\frac{R}{K}(P+2 \pi \Delta \gamma R) .
$$

It is obvious that upon application of a negative load, $P_{\mathrm{c}(\mathrm{DMT})}$ is given by:

$$
P_{\mathrm{c}(\mathrm{DMT})}=-2 \pi \Delta \gamma R,
$$

and the contact radius is zero, which means the two surfaces separate at that point. Therefore, $P_{\mathrm{c}(\mathrm{DMT})}$ is the critical force required to separate the two spheres, i.e. the pull-off force. The model also gives a particular value for the contact radius at a zero load as:

$$
a_{0(\mathrm{DMT})}=\left(2 \pi \Delta \gamma R^{2} / K\right)^{1 / 3} .
$$

The adhesion contact between a solid rigid sphere and an elastic half space has been described by Johnson, Kendall and Roberts (JKR), which leads to the famous JKR theory [11]. They found that the contact radius, $a_{\mathrm{JKR}}$, for a rigid sphere in contact with a compliant elastic half space, was related to the work of adhesion, $\Delta \gamma$, as:

$$
a_{\mathrm{JKR}}^{3}=\frac{R}{K}\left\{P+3 \pi \Delta \gamma R+\left[6 \pi \Delta \gamma R P+(3 \pi \Delta \gamma R)^{2}\right]^{1 / 2}\right\} .
$$

According to the JKR theory, upon application of the negative load, separation of the surfaces would occur when the external force, $P_{\mathrm{c}(\mathrm{JKR})}$, was applied such that

$$
P_{\mathrm{c}(\mathrm{JKR})}=-\frac{3}{2} \pi \Delta \gamma R \text {. }
$$

The model also predicts a particular value for the contact radius at a zero load as:

$$
a_{0(\mathrm{JKR})}=\left(\frac{6 \pi \Delta \gamma R^{2}}{K}\right)^{1 / 3} .
$$

It should be noted that the pull-off force $P_{\mathrm{c}(\mathrm{JKR})}$ is independent of the elastic modulus and depends only on the equivalent radius of curvature and work of adhesion. So equation (7) should apply equally well to a rigid sphere, but this would be contradictory to equation (4). The apparent discrepancy led to a heated debate and later, following the analysis of Tabor [12], Muller et al. [13] pointed out that 
the two theories represented the opposite extremes of a dimensionless parameter $\mu$ given by

$$
\mu=\left(\frac{R \Delta \gamma^{2}}{E^{* 2} \varepsilon^{3}}\right)^{1 / 3},
$$

where $\varepsilon$ is the equilibrium spacing in the Lennard-Jones potential and

$$
E^{*}=\left(\frac{1-v_{1}^{2}}{E_{1}}+\frac{1-v_{2}^{2}}{E_{2}}\right)^{-1}
$$

is the combined elastic modulus. The significance of the Tabor number $\mu$ in the contact theory, especially at the nanoscale, has attracted attention from many researchers (see Ref. [4]). $\mu$ can be interpreted as the ratio of elastic deformation resulting from adhesion to the effective range of surface forces. Another dimensionless number, called transition parameter $\lambda$, was introduced by Maugis [14] and the transition parameter is related to $\mu$ by $\lambda=1.157 \mu$. For an appropriate use of the adhesion models, an adhesion map has been constructed by Johnson and Greenwood [15] using the Dugdale force-separation law with two parameters: $\mu$ (or $\lambda$ ) and $\bar{P}$, where the dimensionless load parameter $\bar{P}$ is the ratio of the applied load to the adhesion force. The JKR theory is applicable to large radius, compliant solids $(\mu>5)$ and the DMT theory applies to small radius, rigid solids $(\mu<0.1)$. Physically, the JKR theory accounts for adhesion forces within the expanded area of contact only, whereas the DMT theory accounts for adhesion forces just outside the contact circle only, which are depicted in Fig. 1b and Fig. 1c, respectively. Table 1 presents the major assumptions and limitations inherent to each theory.

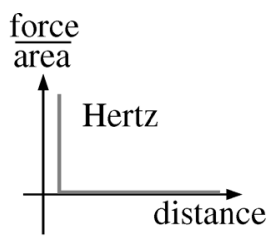

(a)

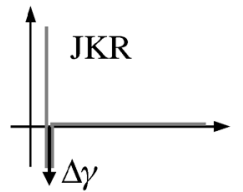

(b)

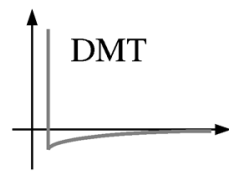

(c)

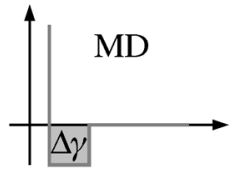

(d)

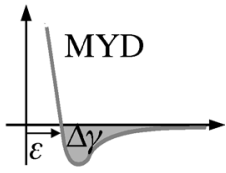

(e)

Figure 1. Interactive forces for the Hertz, JKR, DMT, MD and MYD models. The Hertz model does not consider the adhesion in contact. The JKR model includes only a short-range adhesion in the contact area, actually it is a $\delta$ function with the work of adhesion $\Delta \gamma$. The DMT model shows a longrange surface force which acts outside the radius of the circle of contact. The MD model considers the Dugdale (square well) potential to describe attractive forces. The MYD model, however, includes both the short-range and long-range forces acting both inside and outside the circle of contact, just like the Lennard-Jones potential. 
Table 1.

Comparison of the various contact theories

\begin{tabular}{|c|c|c|}
\hline Theory & Assumptions & Limitations \\
\hline $\begin{array}{l}\text { Hertz } \\
\text { (Fig. 1a) }\end{array}$ & No surface forces & $\begin{array}{l}\text { Not valid for low loads if surface forces } \\
\text { are present }\end{array}$ \\
\hline $\begin{array}{l}\text { JKR } \\
\text { (Fig. 1b) }\end{array}$ & $\begin{array}{l}\text { Short-ranged surface forces act only in- } \\
\text { side contact area } \\
\text { Contact geometry allowed to deform }\end{array}$ & $\begin{array}{l}\text { May underestimate loading due to surface } \\
\text { forces } \\
\text { Applies to high } \lambda \text { systems only }\end{array}$ \\
\hline $\begin{array}{l}\text { DMT } \\
\text { (Fig. 1c) }\end{array}$ & $\begin{array}{l}\text { Long-ranged surface forces act only out- } \\
\text { side contact area }\end{array}$ & $\begin{array}{l}\text { May underestimate contact area due to } \\
\text { restricted geometry } \\
\text { Applies to low } \lambda \text { systems only }\end{array}$ \\
\hline $\begin{array}{l}\text { Maugis- } \\
\text { Dugdale }\end{array}$ & $\begin{array}{l}\text { Periphery of tip-sample interface mod- } \\
\text { eled as a crack that fails at its theoretical } \\
\text { strength }\end{array}$ & $\begin{array}{l}\text { Analytical solution, but parametric equa- } \\
\text { tions } \\
\text { Applies to all values of } \lambda\end{array}$ \\
\hline
\end{tabular}

\section{COMPARISON OF THE DMT AND JKR MODELS WITH THE HERTZ MODEL}

To compare the JKR model with the Hertz model, especially the influence of the adhesion force on the contact radius, it is convenient to make equation (6) dimensionless as

$$
\frac{a_{\mathrm{JKR}}}{a_{\mathrm{H}}}=\left\{1+\frac{3 \pi \Delta \gamma R}{P}+\left[\frac{6 \pi \Delta \gamma R}{P}+\left(\frac{3 \pi \Delta \gamma R}{P}\right)^{2}\right]^{1 / 2}\right\}^{1 / 3},
$$

where the term on left-hand side is always larger than (or equal to) unity as expected, and can be expressed in the dimensionless load parameter $\bar{P}=P /(\pi \Delta \gamma R)$ as

$$
\frac{a_{\mathrm{JKR}}}{a_{\mathrm{H}}}=\left\{1+\frac{3}{\bar{P}}+\left[\frac{6}{\bar{P}}+\left(\frac{3}{\bar{P}}\right)^{2}\right]^{1 / 2}\right\}^{1 / 3} \text {. }
$$

It seems that the dimensionless load parameter $\bar{P}$ dominates the change in the contact radius in the JKR model. The radius $a_{\mathrm{JKR}}$ increases with the work of adhesion $\Delta \gamma$ and with decreasing applied load. This has been pointed out in Yang's work [16]. However, there has been no such analysis for the DMT model. Equations (3) and (1) give

$$
\frac{a_{\mathrm{DMT}}}{a_{\mathrm{H}}}=\left(1+\frac{2}{\bar{P}}\right)^{1 / 3} .
$$

From equation (11), it seems that the radius also increases with the work of adhesion and with decreasing applied load, as in the JKR model.

Figure 2 shows that the indentation radius is controlled by the work of adhesion when the load is less than $10^{-3} \mathrm{mN}$. For a nano-indentation tip radius $1 \mu \mathrm{m}$, and the work of adhesion between the tip and the thin film, $100 \mathrm{~mJ} / \mathrm{m}^{2}$, the ratio $a_{\mathrm{JKR}} / a_{\mathrm{H}}$ in the JKR model decreases from 12.4 at the applied load of $1 \mathrm{nN}$ to 1.5 at a load 


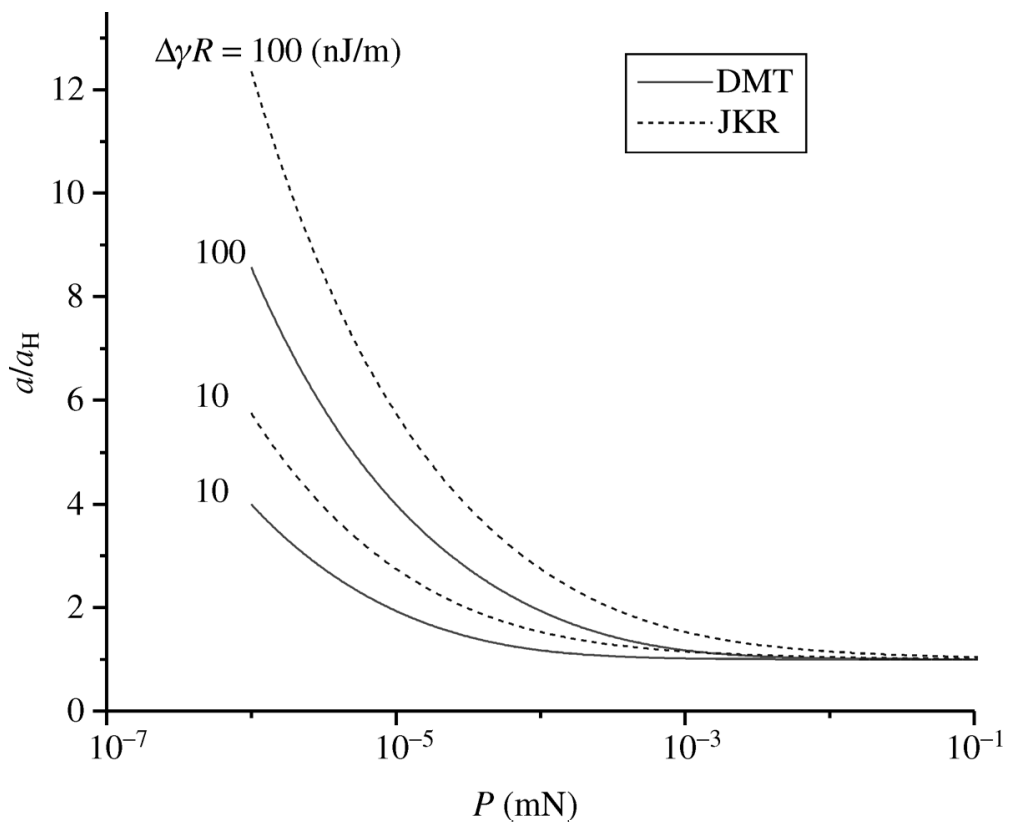

Figure 2. Influence of work of adhesion $\Delta \gamma$ and applied load $P$ on nano-indentation tip radius ratio in both JKR and DMT models.

of $1 \mu \mathrm{N}$, and in the DMT model, the ratio $a_{\mathrm{DMT}} / a_{\mathrm{H}}$ decreases also from 8.6 at a load of $1 \mathrm{nN}$ to 1.2 at a load of $1 \mu \mathrm{N}$. Gradually, the ratios for both models reach the value of 1 as the load approaches $1 \mathrm{mN}$. At small loads, the radius increases sharply with the work of adhesion. It proves that under small loads, the contact radius is dominated by the work of adhesion and the indenter tip radius $R$. Under large loads, the work of adhesion is negligible compared to the strain energy, which controls the deformation of the specimen surface. In conclusion, in both JKR and DMT models, for a small load and a large indenter size, the change in the work of adhesion controls the contact radius between the indenter and the substrate. In contrast, for a large load and small size of the indenter, the elastic deformation dominates.

Also, from equations (10) and (11), it can be seen that the dimensionless load parameter, $\bar{P}=P /(\pi \Delta \gamma R)$, independently controls the influence of the work of adhesion. In Fig. 3, when the value of $\Delta \gamma R / P$ is less than 0.1 , the influence of the work of adhesion is still insignificant. With the value of $\Delta \gamma R / P$ increasing to $10^{2}$, the corresponding value of $a / a_{\mathrm{H}}$ is so large that the work of adhesion must not be ignored and would play the main role in the process of contact.

It should be noted that, in Figs 2 and 3, with the same parameter $\Delta \gamma R$, the values denoted by the dashed curve are always larger than that of the solid curve at most sites, which means that the influence of the work of adhesion in the JKR model is more prominent than in the DMT model. Actually, dividing equation (6) by 


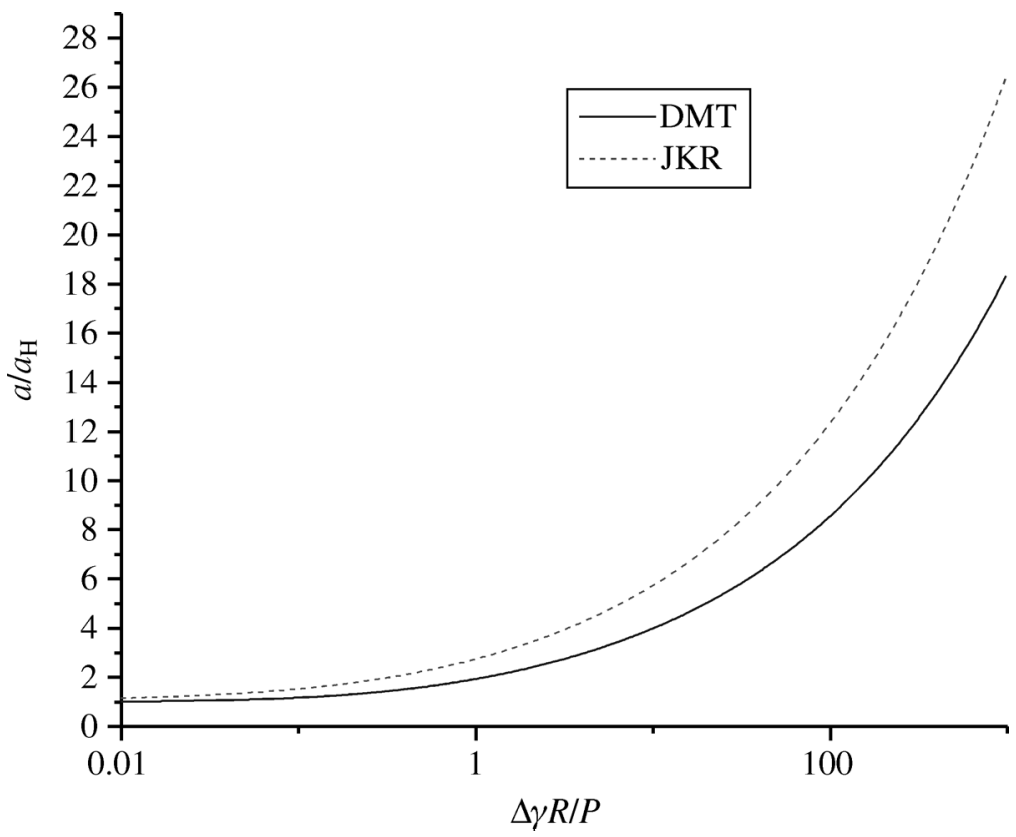

Figure 3. Influence of dimensionless number $\Delta \gamma R / P$ on nano-indentation tip radius ratio in both JKR and DMT models.

equation (3), the ratio between $a_{\mathrm{JKR}}$ and $a_{\mathrm{DMT}}$ is given as:

$$
\frac{a_{\mathrm{JKR}}}{a_{\mathrm{DMT}}}=\left\{\frac{1+3 / \bar{P}+\left[6 / \bar{P}+(3 / \bar{P})^{2}\right]^{1 / 2}}{1+2 / \bar{P}}\right\}^{1 / 3} .
$$

From equation (12) and Figs 4 and 5, it seems that with increasing work of adhesion, the JKR model has more influence on the contact radius. Also, an ultimate value of $a_{\mathrm{JKR}} / a_{\mathrm{DMT}}, \sqrt[3]{3}$, can be deduced with the decrease of applied load.

In the above discussion about the difference in the JKR and DMT models, the effect of material properties such as the elastic modulus was not considered. Actually, equations (1), (3) and (6), respectively, can be recast into:

$$
\begin{aligned}
P_{\mathrm{H}} & =\frac{K a^{3}}{R}, \\
P_{\mathrm{DMT}} & =\frac{K a^{3}}{R}-2 \pi \Delta \gamma R, \\
P_{\mathrm{JKR}} & =\frac{K a^{3}}{R}-\left(6 \pi \Delta \gamma K a^{3}\right)^{1 / 2} .
\end{aligned}
$$

Then the additional terms $P_{\mathrm{A}(\mathrm{DMT})}$ and $P_{\mathrm{A}(\mathrm{JKR})}$ compared with the Hertz model in equation (13) are given by:

$$
\begin{aligned}
P_{\mathrm{A}(\mathrm{DMT})} & =2 \pi \Delta \gamma R, \\
P_{\mathrm{A}(\mathrm{JKR})} & =\left(6 \pi \Delta \gamma K a^{3}\right)^{1 / 2} .
\end{aligned}
$$




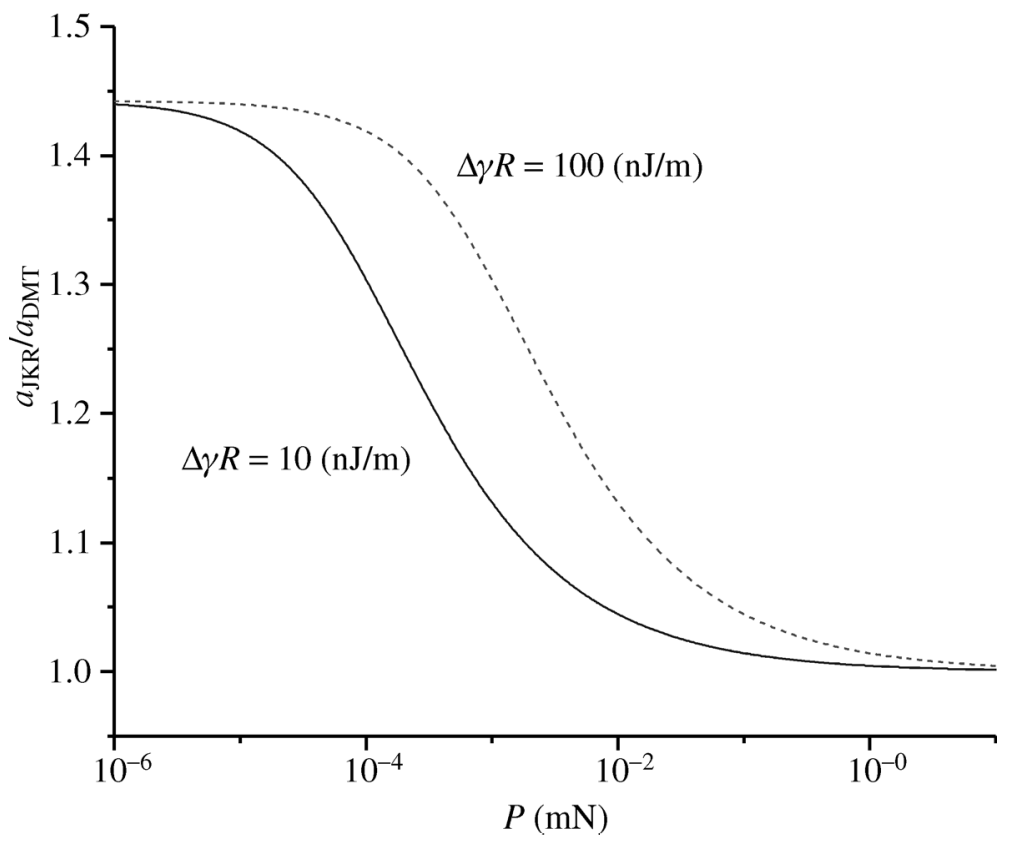

Figure 4. The ratio of $a_{\mathrm{JKR}}$ to $a_{\mathrm{DMT}} v s$. the load $P$. Under the same load $P$ and within the region $10^{-4}-10^{-1} \mathrm{mN}$, the ratio $a_{\mathrm{JKR}} / a_{\mathrm{DMT}}$ increases appreciably when $\Delta \gamma R$ changes from $10 \mathrm{~nJ} / \mathrm{m}$ to $100 \mathrm{~nJ} / \mathrm{m}$, which shows that the work of adhesion has more influence on the JKR model.

Then the ratios between $P_{\mathrm{A}(\mathrm{DMT})}$ and $P_{\mathrm{H}}$, as well as between $P_{\mathrm{A}(\mathrm{JKR})}$ and $P_{\mathrm{H}}$ are given by:

$$
\begin{aligned}
& \frac{P_{\mathrm{A}(\mathrm{DMT})}}{P_{\mathrm{H}}}=\left(\frac{R}{a}\right)^{2} \frac{2 \pi \Delta \gamma}{a K}, \\
& \frac{P_{\mathrm{A}(\mathrm{JKR})}}{P_{\mathrm{H}}}=\frac{R}{a}\left(\frac{6 \pi \Delta \gamma}{a K}\right)^{1 / 2} .
\end{aligned}
$$

Here, the quantity $a / R$ is proportional to the indentation strain [1]. The value of $a K$ determinates whether or not the adhesion force is significant for a particular contact. As $a K$ becomes large, the additional term $P_{A}$ becomes small. In the case of small $K$, the additional term is significant for very compliant surfaces even when the contact radius is large. Also, as the DMT model describes the contact by the Bradley theory [9], which considers that all contacting bodies were rigid, it is appropriate to use the DMT model if the modulus $K$ is relatively large. In the case that the material is compliant, the JKR theory is suitable because the JKR model deals with the contact between a rigid sphere and an elastic compliant half space [11]. Actually, the JKR model is valid for low modulus, high surface energy materials and large indenters, whereas the DMT model is valid for more rigid materials with lower surface energies and for smaller indenters. It should be noted that in equations (18) 


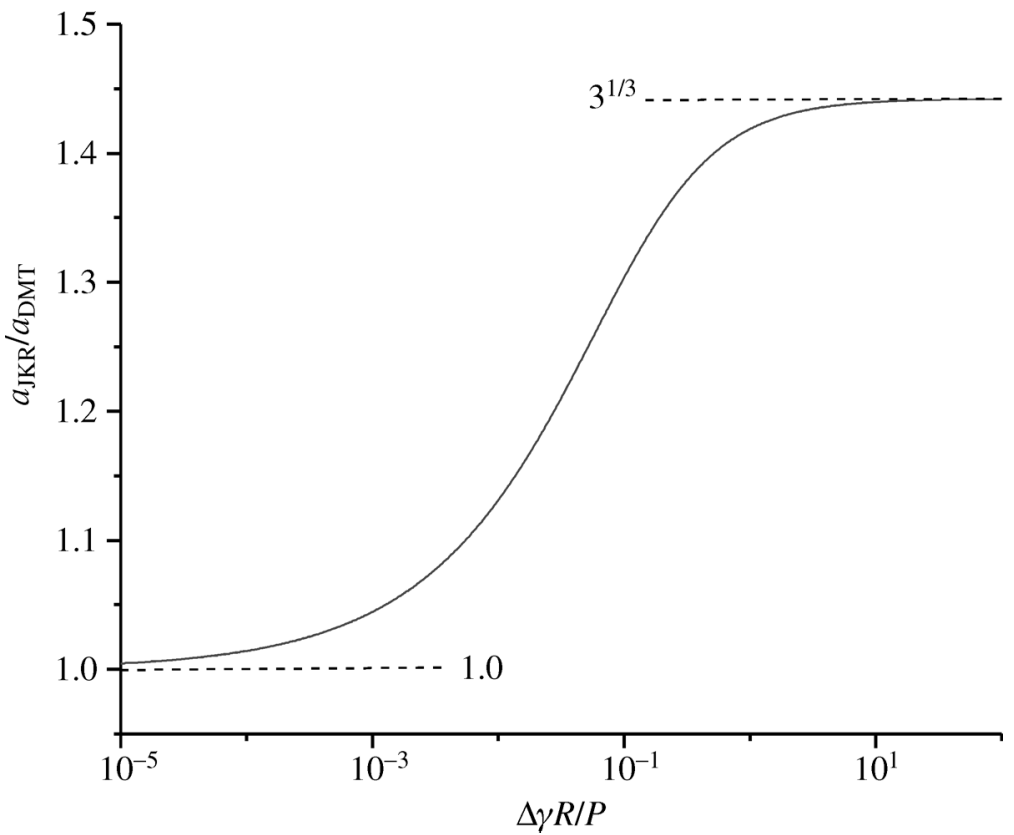

Figure 5. The ratio of $a_{\mathrm{JKR}}$ to $a_{\mathrm{DMT}} v s$. the dimensionless number $\Delta \gamma R / P$. When the value of $\Delta \gamma R / P$ increases, the ratio $a_{\mathrm{JKR}} / a_{\mathrm{DMT}}$ increases from the lower critical value to the upper one. The lower critical value, 1.0, shows that when surface energy can be ignored, the contact radii in both JKR and DMT models are similar. Actually they are similar to what the Hertz model predicted. The upper critical value, $3^{1 / 3}$, however, shows the difference in contact radii between the JKR and the DMT models.

and (19), the ratios, in fact, express the ratios between the surface energy and the elastic energy, which has the similar scale as the Tabor parameter in equation (9).

\section{COMPARISON OF THE MD MODEL WITH THE HERTZ MODEL}

The differences in the contact radii and separation forces between the JKR model and the DMT model which were denoted in equations (4), (5), (7) and (8) have caused some debate $[17,18]$. As a result, Muller et al. proposed the Muller, Yushchenko and Derjaguin (MYD) model $[13,19]$ and described the adhesion force between the indenter tip and the sample by a pair-wise summation of the molecules via a Lennard-Jones potential. By using the Dugdale potential, Maugis [14] proposed the Maugis-Dugdale (MD) model in 1992. It was found that the MD model was the general case in describing the contact and both the JKR and DMT models were special cases. The transition between the JKR and DMT models was investigated by Maugis and Gauthier-Manuel [20], who used the 'Dugdale' (square well) potential to describe the attractive forces between contacting spheres 


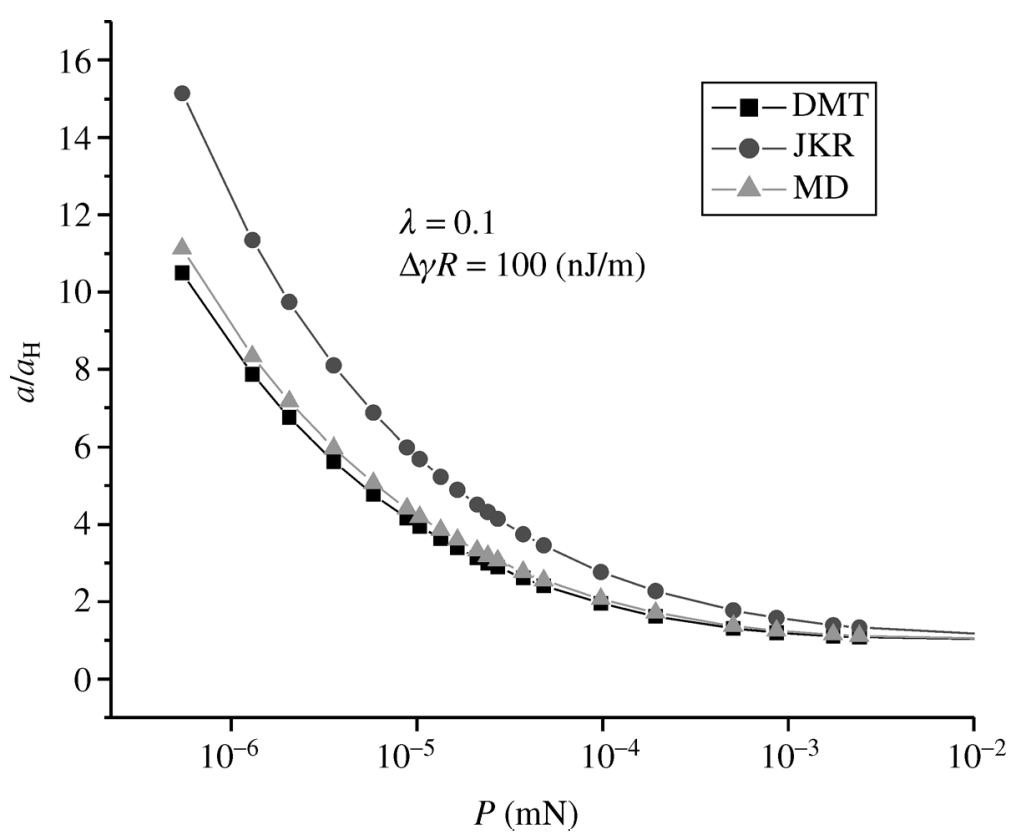

Figure 6. Effect of applied load $P$ on nano-indentation tip radius ratio in JKR, DMT and MD models when $\lambda=0.1$ and $\Delta \gamma R=100 \mathrm{~nJ} / \mathrm{m}$.

and obtained the following equations:

$$
\begin{aligned}
& \frac{1}{2} \lambda \bar{a}^{2}\left[\sqrt{m^{2}-1}+\left(m^{2}-2\right) \arccos (1 / m)\right] \\
& \quad+\frac{4}{3} \lambda^{2} \bar{a}^{2}\left[\sqrt{m^{2}-1} \arccos (1 / m)-m+1\right]=1, \\
& \bar{P}=\bar{a}^{3}-\lambda \bar{a}^{2}\left[\sqrt{m^{2}-1}+m^{2} \arccos (1 / m)\right],
\end{aligned}
$$

where $\bar{a} / a=\left(K / \pi \Delta \gamma R^{2}\right)^{1 / 3}, \bar{c} / c=\left(K / \pi \Delta \gamma R^{2}\right)^{1 / 3}, m=c / a, \bar{P}=P /(\pi \Delta \gamma R)$ and $c$ is the outer radius given as $c=a+0.971 \varepsilon$, where $a$ is the radius of the contact circle, and $\varepsilon$ is the equilibrium spacing in the Lennard-Jones potential.

It is difficult in the MD model to obtain the expression relating only $a$ and $P$ as was the case in JKR and DMT models, because there is another parameter $c$ that varies with $\lambda$. Therefore, it is necessary to use numerical calculations to obtain the solution. From Figs 6-8, the conclusion about the transition between the JKR model and the DMT model is verified. When $\lambda=0.1$, the curve for the MD model approaches the curve due to the DMT model and in the case $\lambda=5$, the curve for the MD model approximately coincides with that for the JKR model. With a value of $\lambda$ between 0.1 and $5, \lambda=1.5$, the curve is in-between those for the other two. So, it can be clearly seen that the JKR and DMT models are two special cases of the MD model. 


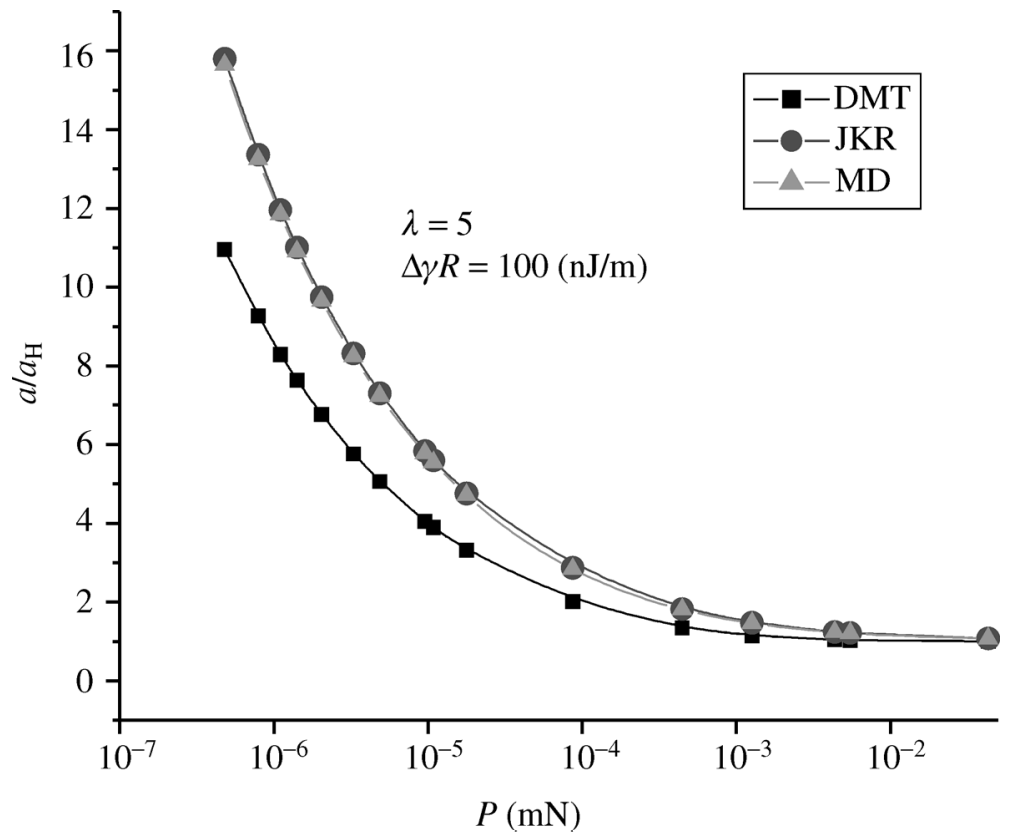

Figure 7. Effect of applied load $P$ on nano-indentation tip radius ratio in JKR, DMT and MD models when $\lambda=5$ and $\Delta \gamma R=100 \mathrm{~nJ} / \mathrm{m}$.

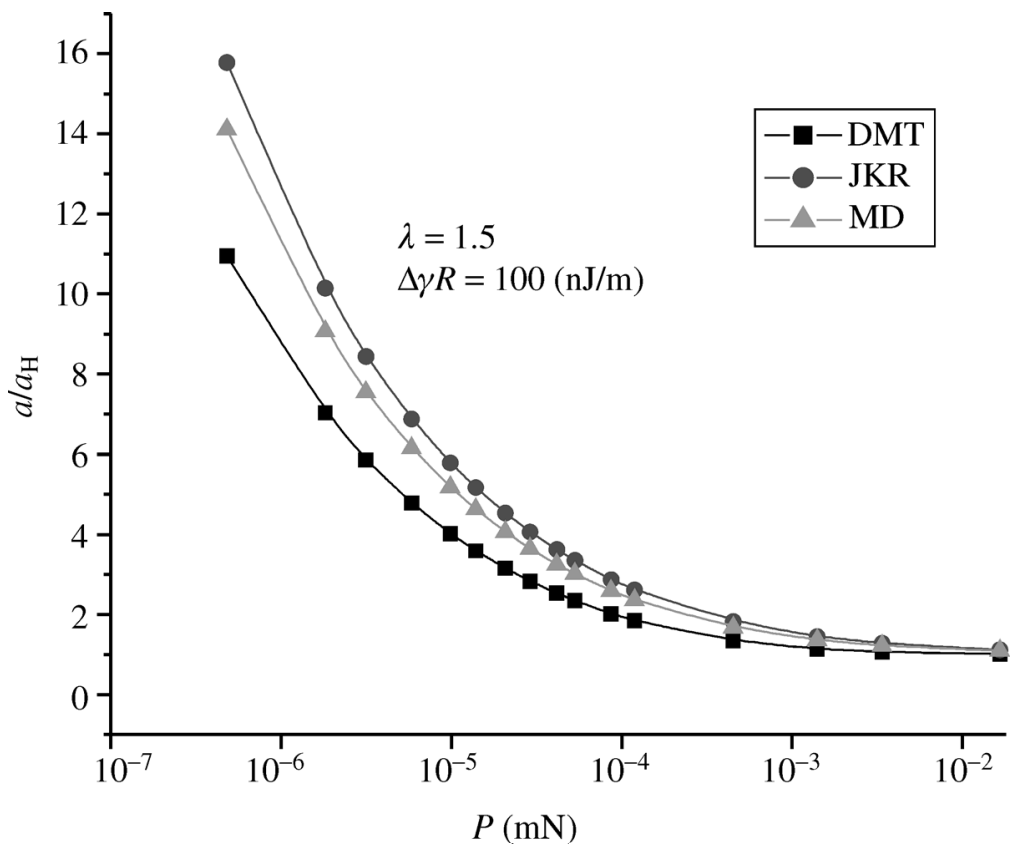

Figure 8. Effect of applied load $P$ on nano-indentation tip radius ratio in JKR, DMT and MD models when $\lambda=1.5$ and $\Delta \gamma R=100 \mathrm{~nJ} / \mathrm{m}$. 


\section{CONCLUSION}

We analyzed the influences of the dimensionless load parameter, $\bar{P}=P /(\pi \Delta \gamma R)$, and the transition parameter, $\lambda$, on the nano-contact area, and we also validated the importance of the work of adhesion through comparison of the JKR, DMT and MD models with the Hertz model. The major conclusions drawn are given below:

(1) With a small applied load, the work of adhesion does play a key role in the micro and/or nano-scale contact and must be taken into account. The difference between the JKR and DMT models is also discussed and the origin of the difference is analyzed in-depth.

(2) The numerical examples show that both the JKR and DMT models are the upper and lower limits of the MD model, respectively. So, the MD model is the general case and can deal with most materials in contact. A large body of experiment results [21] showed that, in general, the AFM measurements were mostly located in the regime of the MD model. So considering the accuracy of the experiments, it is more suitable to use the MD model to deal with the problem. As the MD model is not so concise and the solutions of the equations are cumbersome, it would be much helpful to find some rapid and simple method to determine the parameters which describe the contact. Fortunately, some progress has been made [22, 23].

The influence of the work of adhesion on nano-scale plastic deformation has to be considered for a nano-indentation characterization and modeling. Considering the influence of the work of adhesion, the nano-indentation hardness for a fully plastic contact can be expressed as [24]:

$$
H_{\text {adhesion }}=H\left(1+\frac{2}{\bar{P}}\right) \text {, }
$$

where $H=P / \pi a^{2}$ is the hardness, and $P$ again is the applied load and $a$ is the plastic contact area. Equation (22) shows the influence of the dimensionless load parameter on the hardness. In other words, the influence of the work of adhesion is strong when the applied load is light. Surface roughness has also to be considered at the nano-scale in the future work.

\section{Acknowledgements}

This research was sponsored by the Distinguished Young Scholar Fund of NSFC (Grant No. 10225209), key project from the Chinese Academy of Sciences (Grant No. KJCX2-SW-L2) and National ‘973’ Project (No. G1999033103).

\section{REFERENCES}

1. A. C. Fischer-Cripps, Nano-indentation. Springer, Berlin (2002).

2. N. A. Burnham, S. P. Baker and H. M. Pollock, J. Mater. Res. 15, 2006 (2002). 
3. H. Hertz, J. Reine Angew. Math. 92, 156 (1881).

4. K. L. Johnson, Contact Mechanics. Cambridge University Press, Cambridge (1987).

5. Y.-P., Zhao, L. S. Wang and T. X. Yu, J. Adhesion Sci. Technol. 17, 519 (2003).

6. D. S. Rimai, L. P. DeMejo and K. L. Mittal (Eds), Fundamentals of Adhesion and Interfaces. VSP, Utrecht (1995).

7. F. Restagno and J. Crassous, Phys. Rev. E 65, 042301 (2002).

8. D. Erts, Appl. Surface Sci. 188, 460 (2002).

9. R. S. Bradley, Phil. Mag. 13, 853 (1932).

10. B. V. Derjaguin, V. M. Muller and Yu. P. Toporov, J. Colloid Interface Sci. 53, 314 (1975).

11. K. L. Johnson, K. Kendall and A. D. Roberts, Proc. R. Soc. London A 324, 301 (1971).

12. D. Tabor, J. Colloid Interface Sci. 58, 2 (1977).

13. V. M. Muller, V. S. Yushchenko and B. V. Derjaguin, J. Colloid Interface Sci, 77, 91 (1980).

14. D. Maugis, J. Colloid Interface Sci. 150, 243 (1992).

15. K. L. Johnson and J. A. Greenwood, J. Colloid Interface Sci. 192, 326 (1997).

16. F. Q. Yang, Appl. Phys. Lett. 80, 959 (2002).

17. B. V. Derjaguin, V. M. Muller and Yu. P. Toporov, J. Colloid Interface Sci. 67, 378 (1978).

18. D. Tabor, J. Colloid Interface Sci. 67, 380 (1978).

19. V. M. Muller, V. S. Yushchenko and B. V. Derjaguin, J. Colloids Interface Sci. 92, 92 (1983).

20. D. Maugis and B. Gauthier-Manuel, J. Adhesion Sci. Technol. 8, 1311 (1994).

21. B. Cappella and G. Dietler, Surface Sci. Rep. 34, 1 (1999).

22. R. W. Carpick, D. F. Ogletree and M. Salmeron, J. Colloid Interface Sci. 211, 395 (1999).

23. E. Barthel, J. Colloid Interface Sci. 200, 7 (1998).

24. S. K. R. Chowdhury and H. M. Pollock, Wear 66, 307 (1981).

\section{APPENDIX}

List of symbols

a contact radius

$\bar{a} \quad a\left(K / \pi \Delta \gamma R^{2}\right)^{1 / 3}$

$c \quad$ outer contact radius

$\bar{c} \quad c\left(K / \pi \Delta \gamma R^{2}\right)^{1 / 3}$

E $\quad$ Elastic modulus

$E^{*} \quad\left(\frac{1-v_{1}^{2}}{E_{1}}+\frac{1-v_{2}^{2}}{E_{2}}\right)^{-1}$, combined elastic modulus

$K \quad \frac{4}{3}\left(\frac{1-v_{1}^{2}}{E_{1}}+\frac{1-v_{2}^{2}}{E_{2}}\right)^{-1}$, equivalent elastic modulus

$m \quad c / a$

$P \quad$ applied load

$P_{\mathrm{A}} \quad$ adhesion force

$P_{\mathrm{c}} \quad$ pull-off force

$\bar{P} \quad P /(\pi \Delta \gamma R)$, dimensionless load parameter

$R \quad$ radius of sphere

$\Delta \gamma \quad$ work of adhesion

$\varepsilon \quad$ intermolecular spacing

$\lambda \quad 1.157 \mu$, transition parameter 
$\mu \quad\left(\frac{R \Delta \gamma^{2}}{E^{* 2} \varepsilon^{3}}\right)^{1 / 3}$, Tabor number

$v$ Poisson's ratio 